American Journal of Nursing Science
2021; 10(1): $64-70$
http://www.sciencepublishinggroup.com/j/ajns
doi: $10.11648 /$ j.ajns.20211001.22
ISSN: $2328-5745$ (Print); ISSN: $2328-5753$ (Online)

\title{
Meta-analysis of Risk Factors for Mortality in Patients with Acinetobacter Baumannii Bloodstream Infection
}

\author{
Xu Han, Haiyan Huang*, Weitao Lv, Wanxian Lu \\ Department of Intensive Care Unit, The First Affiliated Hospital of Jinan University, Guangzhou, China \\ Email address: \\ 18002281696@163.com (Xu Han),527917830@qq.com (Haiyan Huang), 13570035005@139.com (Weitao Lv), \\ wanxian63@126.com (Wanxian Lu) \\ *Corresponding author
}

\section{To cite this article:}

Xu Han, Haiyan Huang, Weitao Lv, Wanxian Lu. Meta-analysis of Risk Factors for Mortality in Patients with Acinetobacter Baumannii Bloodstream Infection. American Journal of Nursing Science. Vol. 10, No. 1, 2021, pp. 64-70. doi: 10.11648/j.ajns.20211001.22

Received: February 7, 2021; Accepted: February 16, 2021; Published: February 23, 2021

\begin{abstract}
Background: Patients with Acinetobacter baumannii bloodstream infection (ABBI) have a poor prognosis and high mortality. Early identification of risk factors for death in patients with ABBI, and appropriate prevention and control measures are extremely important to reduce the mortality. Objective: To systematically evaluate the risk factors of ABBI, which provide evidence for reducing the incidence and mortality of patients. Methods: The published articles and reference works on prognosis risk factors in patients with ABBI were retrieved from inception to January 2021 in the following databases: PubMed, Web of Science, Embase, CNKI, Wanfang and VIP. Those studies that met the inclusion criteria were perform quality evaluation and data analysis. Meta-analysis was performed by the statistical software Stata13.0. Results: A total of 24 articles were included, including 1203 patients in the death group and 1319 patients in the survival group. Among the 24 included study factors, 15 showed statistically significant differences $(P<0.01)$, including: age $(\mathrm{MD}=0.19,95 \% \mathrm{CI}: 0.08 \sim 0.30, P<0.01)$, APACHE II $(\mathrm{MD}=0.48,95 \% \mathrm{CI}: 0.06 \sim 0.91, P=0.03)$, ICU admission $(\mathrm{OR}=1.91,95 \% \mathrm{CI}: 1.12 \sim 3.26, P=0.02)$, shock $(\mathrm{OR}=4.28,95 \% \mathrm{CI}$ : 1.05 17.49, $P=0.04)$, septic shock $(\mathrm{OR}=8.36,95 \% \mathrm{CI}: 3.71 \sim 18.82, P<0.01)$, renal damage $(\mathrm{OR}=1.57,95 \% \mathrm{CI}: 1.19 \sim 2.07$, $P<0.01)$, diabetes $(\mathrm{OR}=1.31,95 \% \mathrm{CI}: 1.03 \sim 1.68, P=0.03)$, malignant tumor $(\mathrm{OR}=1.56,95 \% \mathrm{CI}: 1.02 \sim 2.37, P=0.04)$, liver disease $(\mathrm{OR}=1.93,95 \% \mathrm{CI}: 1.25 \sim 3.00, P<0.01)$, hormonal used $(\mathrm{OR}=5.46,95 \% \mathrm{CI}: 3.12 \sim 9.56, P<0.01)$, immunosuppression $(\mathrm{OR}=2.73$, 95\% $\mathrm{CI}: 1.82 \sim 4.1, P<0.01)$, mechanical ventilation $(\mathrm{OR}=3.17,95 \% \mathrm{CI}: 2.34 \sim 4.29, P<0.01)$, deep vein catheterization $(\mathrm{OR}=2.02$, 95\%CI: $1.56 \sim 2.61, P<0.01)$, indwelling urinary catheter $(\mathrm{OR}=2.53,95 \% \mathrm{CI}: 1.66 \sim 3.84, P<0.01)$, indwelling gastric tube $(\mathrm{OR}=1.79,95 \% \mathrm{CI}: 1.21 \sim 2.65, P<0.01)$. Conclusion: The severity of the patient's underlying disease, using of hormones and immunosuppressants, and various invasive operations were the main risk factors for high mortality in ABBI patients. It was necessary to focus on older patients, dynamically monitor the APACHE II score, carefully evaluate the effect of hormone and immunosuppressant application, and strictly control the indications of invasive operation or treatment.
\end{abstract}

Keywords: Acinetobacter Baumannii, Bloodstream Infection, Risk Factors, Mortality, Meta-analysis

\section{Introduction}

Acinetobacter baumannii $(\mathrm{AB})$ is a kind of gram-negative bacilli, which has strong acquired drug resistance and transmission ability [1]. It has become one of the important pathogenies of global hospital infection with the incidence rate increasing. In recent years, the incidence of bloodstream infections caused by $\mathrm{AB}$ has been on the rise [2]. About 2\% $5 \%$ of hospital acquired bloodstream infections are caused by $\mathrm{AB}$ [3]. AB bacteremia can significantly prolong the length of hospital stay and increase the cost of treatment. The prognosis of patients is poor and the mortality rate is high. Septicemia or bacteremia caused by $\mathrm{AB}$ is a common cause of death in patients, with a mortality rate of $27 \% \sim 35 \%$ [3, 4]. In the intensive care unit (ICU), the mortality rate of Acinetobacter baumannii bloodstream infection (ABBI) patients reaches $35 \% \sim 50 \%$ [4]. Therefore, early identification of risk factors for death in patients with ABBI, and targeted application of prevention and control measures are extremely important for reducing the mortality of patients. A number of studies [5-10] have been carried out on the risk factors for the death of ABBI patients. However, the risk factors obtained from different 
studies are not the same, and the results are also different. While this study used meta-analysis to conduct the qualitative and quantitative comprehensive analysis of related articles, and systematically evaluate the risk factors of death in ABBI patients. In order to provide an evidence-based basis for guiding the clinical adjustment of treatment and nursing programs and improving the prognosis of patients.

\section{Materials and Methods}

\subsection{Information Retrieval}

The published studies and reference works on mortality risk factors in patients with $\mathrm{ABBI}$ were retrieved from inception to January 2021 in the following databases: PubMed, Web of Science, Embase, CNKI, Wanfang and VIP. The main search terms were [Title/Abstract]: ('Acinetobacter baumannii' OR 'A baumannii' OR 'Acinetobacter') AND ('bacteremia' OR 'bloodstream infection' OR 'blood infection' OR 'septicemia' OR 'sepsis') AND ('mortality' OR 'fatality' OR 'lethality' OR 'prognosis' OR 'predictor' OR 'survival') AND ('risk factors' OR 'influencing factors'). The corresponding Chinese search terms were used for the Chinese databases. In addition, the references identified by this strategy were searched to select relevant articles. Grey article or unpublished data were not searched for this study.

\subsection{Inclusion and Exclusion Criteria}

\subsubsection{Inclusion Criteria}

Studies reporting risk factors of mortality in patients infected with ABBI were included. The cases met the diagnostic criteria of bloodstream infection, and the blood culture suggested Acinetobacter baumannii infection. The mean difference (MD) or odds ratio (OR) value and 95\% confidence intervals (CI) of multivariate analysis or the data that can calculate OR value and $95 \% \mathrm{CI}$ were included in the articles.

\subsubsection{Exclusion Criteria}

Repetitive published article, reviews, systematic reviews, meta-analyses, guidelines, editorials, case reports, animal research, in vitro studies, and research that focused on specific population (such as children or newborns). Studies with research design defects, no controlled studies, article with incomplete statistical data, unable to obtain or value and $95 \%$ CI of risk factors, were also excluded.

\subsection{Evaluation of Study Quality}

Endnote X9 was used for articles management and duplicate checking. Two authors (Xu H. and Haiyan H.) independently screened and reviewed each study manually for eligibility. According to the Newcastle Ottawa scale (NOS) score, the articles meeting the above criteria were assessed with $0-9$ for quality assurance. Studies with a NOS $\geq 6$ were included in this analysis. Any disagreement was resolved by the adjudicating senior authors (Weitao L. and Wanxian L.).

\subsection{Data Extraction and Statistical Methods}

Excel 2016 was used to design a unified document information extraction table, including: study author, publication year, country, design, number of grouped cases, research risk factors, and NOS score. Two authors (Xu $\mathrm{H}$. and Haiyan H.) independently extracted relevant information of the included articles according to the unified table, and then cross-checked. Stata 13.0 were used for statistical analysis. The measurement data used MD and 95\% CI, the count data used OR and $95 \% \mathrm{CI}$, and the inspection level was $\alpha=0.05$. The heterogeneity test was analyzed using the chi-square test. When $\left(P>0.10, I^{2}<50 \%\right)$, the heterogeneity was small, which the fixed effects model (FE) was used. When $\left(P \leq 0.10, I^{2}\right.$ $\geq 50 \%$ ), the random effects model (RE) was used, and briefly analyzed the causes of heterogeneity. Egger's method was used to analyze the publication bias quantitatively.

\section{Results}

\subsection{The Articles Search Results}

A total of 678 articles were retrieved in this study, including: PubMed ( $n=193)$, Web of Science $(n=162)$, Embase $(n=79)$, CNKI ( $n=143)$, Wanfang $(n=71)$, VIP $(n=30)$. After duplicate checking with Endnote X9, 509 articles were remained, and 438 articles were excluded by reading the title and abstract. About 47 studies were excluded by reading the full text, while 24 studies were finally included. Flow diagram of studies identified, excluded, and included were shown in Figure 1.

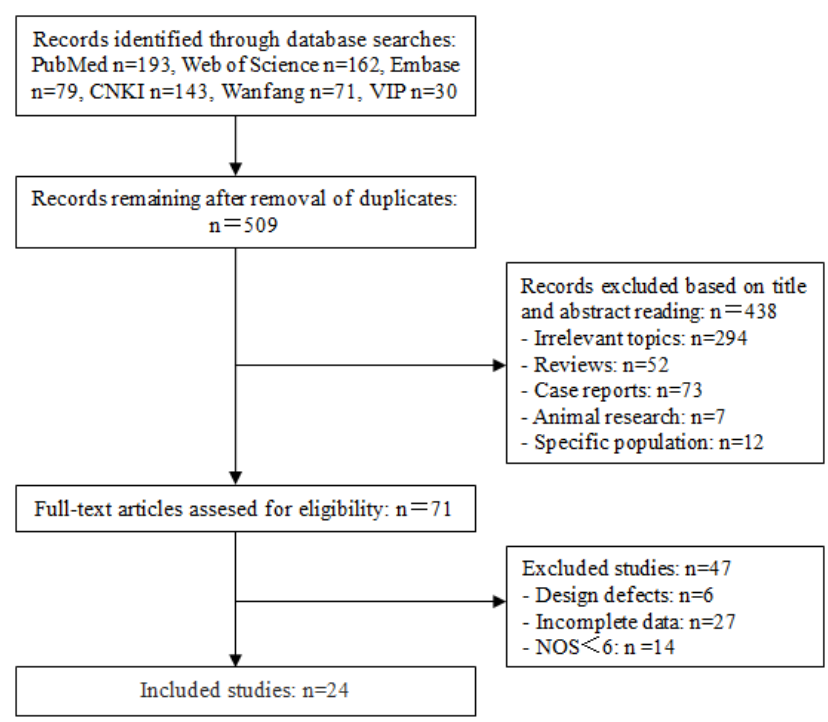

Figure 1. Flow diagram of studies identified, excluded, and included

\subsection{Basic Characteristics of the Included Studies}

The 24 included articles were retrospective or case-control studies. After scoring by NOS standards, there were 10 articles with 8 points, 11 articles with 7 points, and 3 articles with 6 points. The overall quality of those studies was good, which 12 papers published in the last five years. However, the sample size of the included article was small, most studies had 
only dozens cases, as shown in Table 1. A total of 2522

1319 were in the survival group.

patients with ABBI, which 1203 were in the death group and

Table 1. The baseline information of the included studies.

\begin{tabular}{lllllll}
\hline Study Author & Year & Country & Design & Death & Survival & Risk factors \\
\hline Yu K. et al. [5] & 2021 & China & R/MC & 33 & 75 & ABCFHIOPQRS \\
Gu Y. et al. [6] & 2021 & China & Case-control/ SC & 21 & 26 & ACDJKLRSTW \\
Zhou H. et al. [7] & 2019 & China & R/MC & 154 & 120 & ABCDFSTUVW \\
Papadimitriou-O. M. et al. [8] & 2017 & Greece & Case-control/ SC & 51 & 78 & ABCFHJKLMPSTU \\
Ballouz T. et al. [9] & 2017 & Lebanon & R/SC & 58 & 32 & ABEFGHIJKLMPTUVWX \\
Wang, X. et al. [10] & 2017 & China & R/MC & 13 & 27 & ABCDEFKMPQ \\
Yanyan Z. et al. [11] & 2017 & China & R/SC & 40 & 38 & ABCDFHILMTU \\
Zhang Y. et al. [12] & 2017 & China & R/SC & 53 & 95 & ABDFGHILMOPUV \\
Gu Z. et al. [13] & 2016 & China & R/SC & 49 & 73 & ADGHIJKLMNQTVWX \\
Lee YT. et al. [14] & 2016 & China & R/MC & 45 & 72 & ADEV \\
Liu CP. et al. [15] & 2016 & China & R/SC & 106 & 76 & ADFHIJKLMTVW \\
Guo N. et al. [16] & 2016 & China & R/SC & 39 & 48 & ACDFOPQRSTV \\
Freire MP. et al. [17] & 2015 & Brazil & R/SC & 77 & 15 & AFJOS \\
Long Y. et al. [18] & 2015 & China & R/SC & 35 & 17 & ABCEGIKLORST \\
Liu, Q. et al. [4] & 2015 & China & R/SC & 53 & 129 & ABDFJKLMOPSTX \\
Nutman A. et al. [19] & 2014 & Israel & Case-control / SC & 83 & 89 & ADQV \\
Park SY. et al. [20] & 2013 & Korea & R/SC & 42 & 81 & ABDEOPQRSTUVX \\
Kim SY. et al. [21] & 2012 & Korea & Case-control/ SC & 79 & 20 & ABCDHIJKLTUWX \\
Esterly JS. et al. [22] & 2011 & United States & R/SC & 31 & 48 & ABOQV \\
Song JY. et al. [23] & 2011 & Korea & R/SC & 15 & 13 & ABGHJKLMNOQTX \\
Anunnatsiri S. et al. [24] & 2011 & Thailand & R/SC & 34 & 15 & ABDFGHJKUV \\
Routsi C. et al. [25] & 2010 & Greece & R/MC & 44 & 52 & ABDEOPQTUX \\
Tseng YC. et al. [26] & 2007 & Taiwan, China & R/SC & 27 & 29 & FGHJKLNQSUVWX \\
Choi JY. et al. [27] & 2005 & Korea & R/SC & 21 & 51 & ABFOPQRS \\
\hline
\end{tabular}

R, retrospective; SC, single center; MC, multicenter. Among the risk factors: A: sex, B: age, C: APACHE II, D: ICU admission, E: shock, F: septic shock, G: cerebrovascular disease, H: cardiovascular disease, I: hypertension, J: renal damage, K: diabetes, L: malignant tumor, M: lung disease, N: liver disease, O: mixed infection, P: hormonal used, Q: immunosuppression, R: carbapenem antibiotic, S: combined antibiotic, T: mechanical ventilation, U:tracheal intubation/incision, V: deep vein catheterization, W: indwelling urinary catheter, X: indwelling gastric tube.

\subsection{Results of Meta-analysis}

Among the 24 included study factors, 15 showed statistically significant differences $(P<0.01)$. These risk factors included: age, APACHE II, ICU admission, shock, septic shock, renal damage, diabetes, malignant tumor, liver disease, hormonal used, immunosuppression, mechanical ventilation, deep vein catheterization, indwelling urinary catheter, indwelling gastric tube, as shown in Table 2. In the analysis of publication bias, the probability of publication deviation among all factors included in the study were small (Egger's test $P \geq 0.05$, Table 2). Taken the risk factors of septic shock, mechanical ventilation, deep vein catheterization, indwelling urinary catheter as examples to draw forest plots, as shown in Figure 2-5.

Table 2. Heterogeneity test and meta-analysis of risk factors for mortality in patients with ABBI at included.

\begin{tabular}{|c|c|c|c|c|c|c|c|c|c|c|c|}
\hline \multirow[t]{2}{*}{ Type of factor } & \multirow[t]{2}{*}{$\begin{array}{l}\text { No. of } \\
\text { studies }\end{array}$} & \multicolumn{2}{|c|}{$\begin{array}{l}\text { No. of patients in studies } \\
\text { reporting specific data }\end{array}$} & \multicolumn{2}{|c|}{$\begin{array}{l}\text { Heterogeneity } \\
\text { Test }\end{array}$} & \multirow[t]{2}{*}{$\begin{array}{l}\text { Analytical } \\
\text { Model }\end{array}$} & \multirow[t]{2}{*}{$\begin{array}{l}\text { Pooled } \\
\text { OR/MD }\end{array}$} & \multirow[t]{2}{*}{$95 \%$ CI } & \multicolumn{3}{|c|}{$P$ value Egger's test } \\
\hline & & Death/ Total & Survival/ Total & $P$ value & $I^{2}(\%)$ & & & & & $t$ & $P>|t|$ \\
\hline Sex (male/ total) & 23 & $769 / 1227^{\S}$ & $709 / 1213^{\S}$ & 0.58 & 0 & $\mathrm{FE}$ & 0.89 & $0.75 \sim 1.06$ & 0.18 & 0.357 & 0.492 \\
\hline Age & 17 & N/A & N/A & 0.1 & 32 & RE & $0.19^{*}$ & $0.08 \sim 0.30$ & 0.00 & -0.396 & 0.600 \\
\hline APACHE II & 9 & N/A & N/A & $<0.01$ & 75.2 & $\mathrm{RE}$ & $0.48^{*}$ & $0.06 \sim 0.91$ & 0.03 & 3.290 & 0.274 \\
\hline ICU admission & 10 & $386 / 686$ & $414 / 563$ & 0.00 & 72 & RE & 1.91 & $1.12 \sim 3.26$ & 0.02 & 0.912 & 0.617 \\
\hline Shock & 5 & $32 / 114$ & $132 / 196$ & $<0.01$ & 77.4 & $\mathrm{RE}$ & 4.28 & $1.05 \sim 17.49$ & 0.04 & 3.392 & 0.615 \\
\hline Septic shock & 12 & $167 / 697$ & $482 / 769$ & $<0.01$ & 86.6 & $\mathrm{RE}$ & 8.36 & $3.71 \sim 18.82$ & 0.00 & 2.198 & 0.274 \\
\hline Cerebrovascular disease & 10 & $78 / 303$ & $58 / 266$ & 1.69 & 30 & FE & 0.99 & $0.63 \sim 1.55$ & 0.96 & 0.384 & 0.892 \\
\hline Cardiovascular disease & 13 & $217 / 776$ & $158 / 747$ & 0.98 & 0 & $\mathrm{FE}$ & 0.83 & $0.63 \sim 1.09$ & 0.18 & -0.528 & 0.154 \\
\hline Hypertension & 6 & $121 / 395$ & $73 / 260$ & 0.07 & 50 & FE & 1.01 & $0.69 \sim 1.48$ & 0.97 & -3.545 & 0.459 \\
\hline Renal damage & 16 & $111 / 847$ & $195 / 935$ & 0.50 & 0 & FE & 1.57 & $1.19 \sim 2.07$ & 0.00 & 0.603 & 0.242 \\
\hline Diabetes & 16 & $158 / 849$ & $230 / 893$ & 0.18 & 23.8 & FE & 1.31 & $1.03 \sim 1.68$ & 0.03 & 0.588 & 0.506 \\
\hline Malignant tumor & 15 & $158 / 810$ & $210 / 788$ & $<0.01$ & 56.7 & $\mathrm{RE}$ & 1.56 & $1.02 \sim 2.37$ & 0.04 & -1.605 & 0.186 \\
\hline Lung disease & 17 & $136 / 866$ & $150 / 905$ & 0.23 & 19.2 & $\mathrm{FE}$ & 0.98 & $0.75 \sim 1.29$ & 0.91 & -0.599 & 0.424 \\
\hline Liver disease & 9 & $46 / 571$ & $64 / 506$ & 0.27 & 19 & FE & 1.93 & $1.25 \sim 3.00$ & 0.00 & 0.757 & 0.449 \\
\hline Mixed infection & 14 & 299/749 & $295 / 673$ & 0.65 & 0 & FE & 1.16 & $0.92 \sim 1.47$ & 0.20 & -0.301 & 0.568 \\
\hline Hormonal used & 5 & $54 / 179$ & $106 / 205$ & 0.63 & 0 & FE & 5.46 & $3.12 \sim 9.56$ & 0.00 & -1.99 & 0.219 \\
\hline Immunosuppression & 7 & $51 / 308$ & $99 / 316$ & 0.27 & 20.6 & FE & 2.73 & $1.82 \sim 4.10$ & 0.00 & -0.632 & 0.684 \\
\hline Carbapenem antibiotic & 5 & $49 / 153$ & $43 / 94$ & $<0.01$ & 73.2 & $\mathrm{RE}$ & 1.56 & $0.50 \sim 4.84$ & 0.44 & -2.698 & 0.726 \\
\hline
\end{tabular}




\begin{tabular}{|c|c|c|c|c|c|c|c|c|c|c|c|}
\hline \multirow[t]{2}{*}{ Type of factor } & \multirow[t]{2}{*}{$\begin{array}{l}\text { No. of } \\
\text { studies }\end{array}$} & \multicolumn{2}{|c|}{$\begin{array}{l}\text { No. of patients in studies } \\
\text { reporting specific data }\end{array}$} & \multicolumn{2}{|c|}{$\begin{array}{l}\text { Heterogeneity } \\
\text { Test }\end{array}$} & \multirow[t]{2}{*}{$\begin{array}{l}\text { Analytical } \\
\text { Model }\end{array}$} & \multirow[t]{2}{*}{$\begin{array}{l}\text { Pooled } \\
\text { OR/MD } \\
\end{array}$} & \multirow[t]{2}{*}{$95 \% \mathrm{CI}$} & \multicolumn{3}{|c|}{$P$ value Egger's test } \\
\hline & & Death/ Total & Survival/ Total & $P$ value & $I^{2}(\%)$ & & & & & $t$ & $P>|t|$ \\
\hline Combined antibiotic & 9 & $206 / 409$ & $220 / 451$ & 0.02 & 67.9 & $\mathrm{RE}$ & 1.41 & $0.74 \sim 2.70$ & 0.30 & -0.214 & 0.881 \\
\hline Mechanical ventilation & 12 & $362 / 622$ & $452 / 574$ & 0.17 & 28.4 & FE & 3.17 & $2.34 \sim 4.29$ & 0.00 & -0.591 & 0.758 \\
\hline Tracheal intubation/incision & 9 & $208 / 552$ & $179 / 396$ & $<0.01$ & 63.9 & $\mathrm{RE}$ & 1.68 & $0.73 \sim 3.83$ & 0.22 & 2.236 & 0.051 \\
\hline Deep vein catheterization & 12 & $424 / 710$ & $447 / 588$ & 0.37 & 7.3 & FE & 2.02 & $1.56 \sim 2.61$ & 0.00 & 1.351 & 0.165 \\
\hline Indwelling urinary catheter & 7 & $215 / 311$ & $294 / 338$ & 0.76 & 0 & FE & 2.53 & $1.66 \sim 3.84$ & 0.00 & 1.371 & 0.130 \\
\hline Indwelling gastric tube & 7 & $131 / 248$ & $157 / 247$ & 0.91 & 0 & $\mathrm{FE}$ & 1.79 & $1.21 \sim 2.65$ & 0.00 & 0.506 & 0.489 \\
\hline
\end{tabular}

APACHE II: Acute Physiology and Chronic Health Evaluation II score; N/A: not applicable; OR: odds ratio; MD: mean difference; ${ }^{{ }}$sex: male/total; ${ }^{*}$ Pooled MD

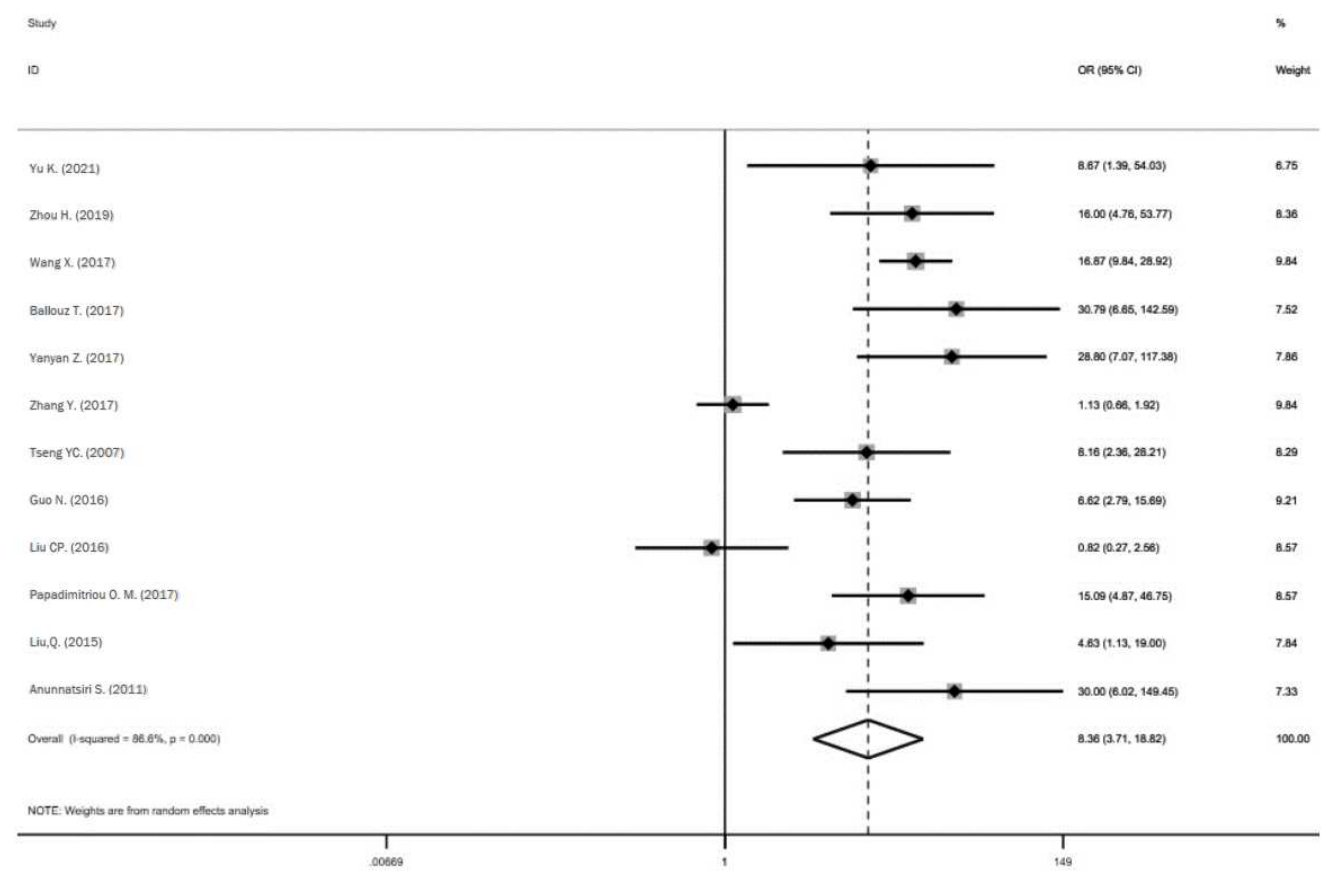

Figure 2. Forest plot and meta-analysis of septic shock.

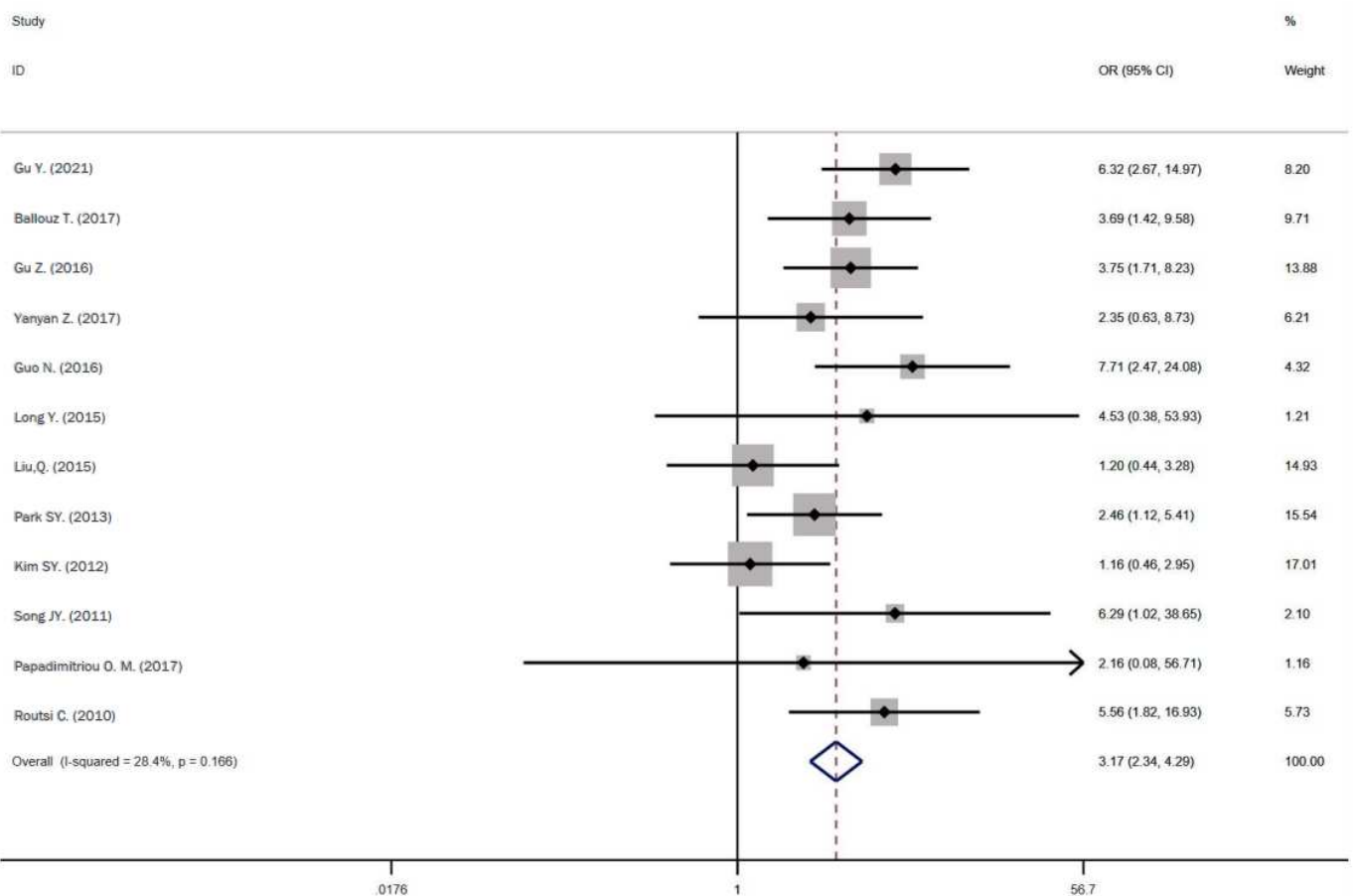

Figure 3. Forest plot and meta-analysis of mechanical ventilation. 


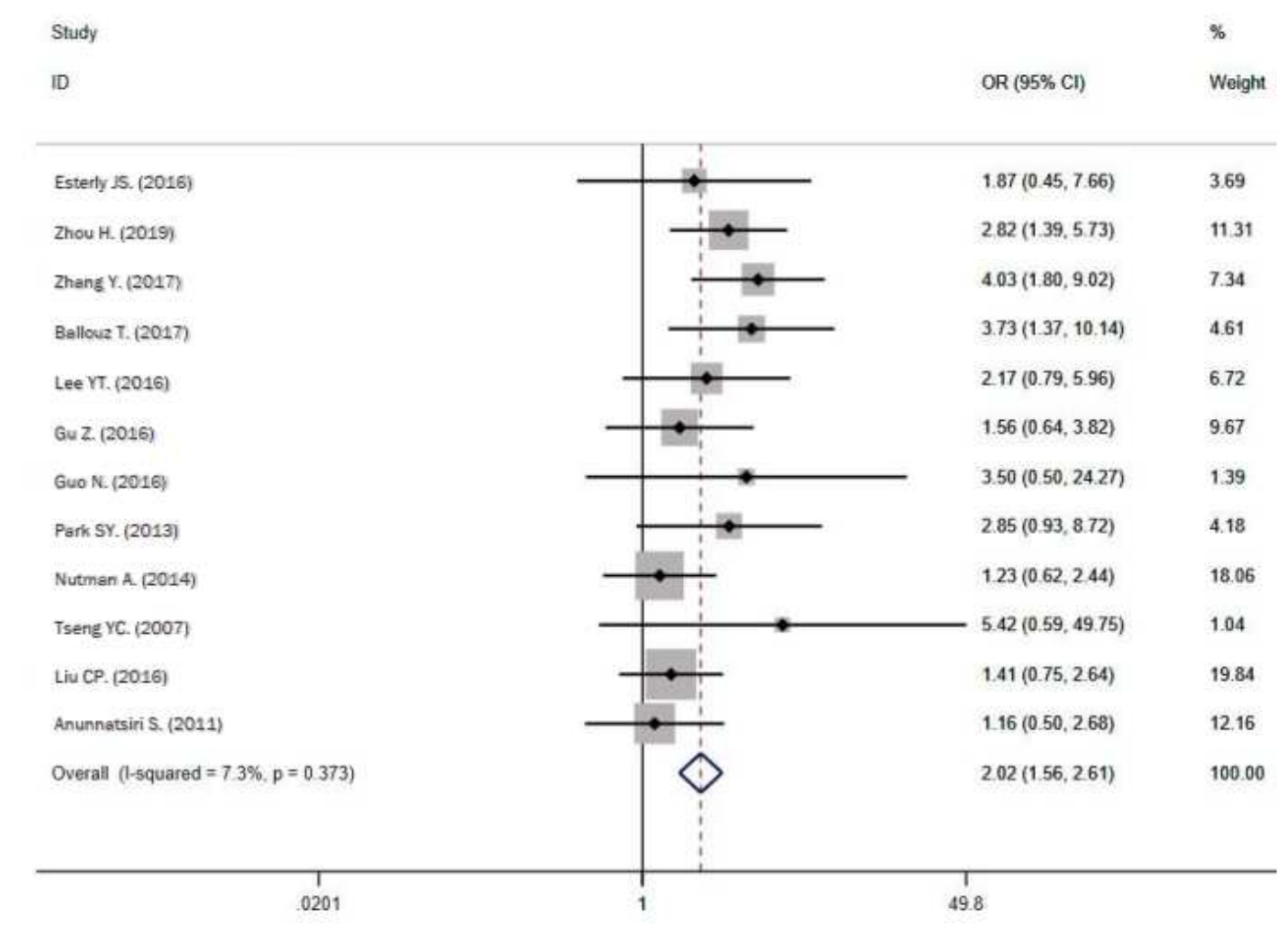

Figure 4. Forest plot and meta-analysis of deep vein catheterization.

Study

ID

OR (95\% Cl)

Weight

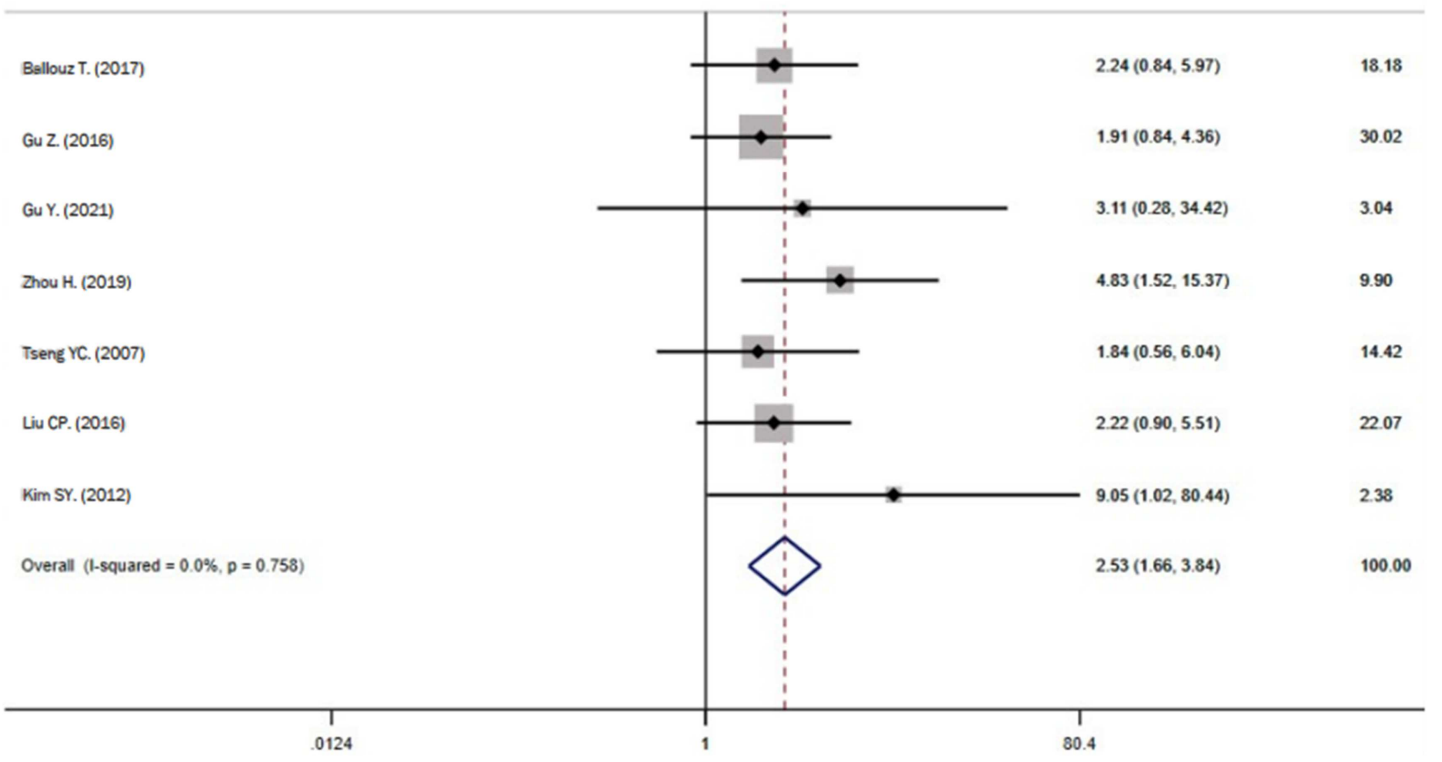

Figure 5. Forest plot and meta-analysis of indwelling urinary catheter.

\section{Discussion}

$\mathrm{AB}$ is one of the main pathogenic bacteria causing nosocomial infections. Because of its strong transmission ability and high drug resistance rate [28], it is widely prevalent in global medical institutions. In recent years, the incidence of bloodstream infections caused by $\mathrm{AB}$ has been on the rise [6]. The prognosis of patients is poor and the mortality rate is high. Early identification of the risk factors for the death of ABBI patients and the targeted adoption of reasonable preventive measures are extremely important for reducing the mortality 
of patients. In this paper, meta-analysis was used to conduct a qualitative and quantitative comprehensive analysis of 24 related articles, and systematically evaluate the risk factors of death in ABBI patients.

The results of this study shown that age, APACHE II, ICU admission, shock, septic shock, renal damage, diabetes, malignant tumor, liver disease, hormonal used, immunosuppression, mechanical ventilation, deep vein catheterization, indwelling urinary catheter, indwelling gastric tube were important risk factors for the death of ABBI patients. The elderly patients might have serious underlying diseases. The patients had poor nutritional status and weakened immune function, leading to poor clinical prognosis. The APACHE II score was a quantitative index for clinical evaluation of the severity of the condition of critically ill patients and the prognosis of the disease. It was currently the most authoritative evaluation system for the condition. The study by Knaus WA et al. [29] showed that after evaluating 5815 critically ill patients, they found that the APACHE II score was directly related to the actual mortality of the patient. The higher of the APACHE II score, the patient's condition was more serious, and the prognosis was worse. The patients admitted to ICU were in critical condition, and they would use a lot of antibiotics and invasive operations during the treatment process. Compared with the patients in the general wards, $A B$ infection had more chances and higher mortality rate [30]. In addition, Xiao D. et al. [31] research suggested that septic shock in the ICU would affect the prognosis of patients infected with $A B$, which was consistent with the results of this study.

The results of this study showed that the use of hormones and immunosuppressants were risk factors for poor prognosis of ABBI. Clinical use of large doses of hormones and immunosuppressants to suppress abnormal immunity, but also inhibit the body's normal humoral immunity and cellular immunity, resulting in reduced immune function of patients, easily to induce infection. It was suggested that the clinical significance of hormone and immunosuppressant should be evaluated carefully. At the same time, invasive mechanical ventilation, deep vein catheterization, indwelling urinary catheter, indwelling gastric tube were the high risk factor for death in patients with ABBI. It has been confirmed in study [32] that mechanical ventilation was a high risk factor for death in patients with ABBI. The probability of microbial colonization would be significantly increased when the patient's airway was open. Similarly, deep vein catheterization, indwelling catheter and indwelling gastric tube all form artificial cavities in patients, which were also exposed to ward environment in the process of treatment and nursing. That could easily lead to the occurrence and development of ABBI, and eventually lead to poor clinical prognosis of patients. Therefore, clinicians should strictly control the indications of invasive procedures and minimize unnecessary catheterization.

There are some limitations in this study: Because the research objects of the included articles came from many countries and regions, there had different levels of infection control and measures, which made some research factors have some heterogeneity. In some studies, the pooled effect size was not calculated due to the inconsistency in the definition of the research factors. Some of the studies had non-standard control selection, comparability between groups, and the determination of exposure factors were not clearly described, which suggested that the original design of these studies needed to be further standardized. Sensitivity analysis was not conducted in this study, and the stability of the results could not be determined. Nevertheless, the overall quality of the literature in this study is relatively high, so the conclusions obtained still have certain clinical reference value. Finally, sensitivity analysis was not carried out in this study, and the stability of the results could not be judged. Nevertheless, the overall quality of those studies were high with NOS standards, so the conclusions still had certain clinical reference value.

\section{Conclusion}

Patients with $\mathrm{ABBI}$ had poor prognosis and high mortality; the risk factors of death were complicated. Identifying controllable related factors and intervening would have a positive effect on reducing the mortality of the patients. In summary, the severity of the patient's age, APACHE II, underlying disease, using of hormones and immunosuppressants, and various invasive operations were the main risk factors for high mortality. In clinical work, it was necessary to focus on older patients, dynamically monitor the APACHE II score, carefully evaluate the effect of hormone and immunosuppressant application, and strictly control the indications of invasive operation or treatment, as to effectively prevent $\mathrm{ABBI}$, improve the prognosis and reduce the mortality in patients.

\section{References}

[1] Kim SY, Cho SI, Bang JH. Risk factors associated with bloodstream infection among patients colonized by multidrug-resistant Acinetobacter baumannii: A 7-year observational study in a general hospital. Am J Infect Control. 2020; 48 (5): 581-583.

[2] Zhang Y, Du M, Johnston JM, et al. Estimating length of stay and inpatient charges attributable to hospital-acquired bloodstream infections. Antimicrob Resist Infect Control. 2020; 9 (1): 137. Published 2020 Aug 18.

[3] Karakonstantis S, Gikas A, Astrinaki E, Kritsotakis EI. Excess mortality due to pandrug-resistant Acinetobacter baumannii infections in hospitalized patients. J Hosp Infect. 2020; 106 (3): 447-453.

[4] Liu Q, Li W, Du X, et al. Risk and Prognostic Factors for Multidrug-Resistant Acinetobacter Baumannii Complex Bacteremia: A Retrospective Study in a Tertiary Hospital of West China. PLoS One. 2015; 10 (6): e0130701. Published 2015 Jun 17.

[5] Yu K, Zeng W, Xu Y, et al. Bloodstream infections caused by ST2 Acinetobacter baumannii: risk factors, antibiotic regimens, and virulence over 6 years period in China. Antimicrob Resist Infect Control. 2021;10 (1): 16. Published 2021 Jan 18. 
[6] Gu Y, Jiang Y, Zhang W, et al. Risk factors and outcomes of bloodstream infections caused by Acinetobacter baumannii: a case-control study. Diagn Microbiol Infect Dis. 2021; 99 (2): 115229 .

[7] Zhou H, Yao Y, Zhu B, et al. Risk factors for acquisition and mortality of multidrug-resistant Acinetobacter baumannii bacteremia: A retrospective study from a Chinese hospital. Medicine (Baltimore). 2019; 98 (13): e14937.

[8] Papadimitriou-Olivgeris M, Fligou F, Spiliopoulou A, et al. Risk factors and predictors of carbapenem-resistant Pseudomonas aeruginosa and Acinetobacter baumannii mortality in critically ill bacteraemic patients over a 6-year period (2010-15): antibiotics do matter. J Med Microbiol. 2017; 66 (8): 1092-1101.

[9] Ballouz T, Aridi J, Afif C, et al. Risk Factors, Clinical Presentation, and Outcome of Acinetobacter baumannii Bacteremia. Front Cell Infect Microbiol. 2017; 7: 156. Published 2017 May 4.

[10] Wang X, Zhang L, Sun A, et al. Acinetobacter baumannii bacteraemia in patients with haematological malignancy: a multicentre retrospective study from the Infection Working Party of Jiangsu Society of Hematology. Eur J Clin Microbiol Infect Dis. 2017; 36 (7): 1073-1081.

[11] Yanyan Z, Wan Z, Jingping Z, et al. The risk factors associated with bloodstream infections caused by multi-drugresistant Acinetobacter baumannii. Chin J Infect Chemother, 2017, 17 (02): 134-139.

[12] Zhang Y, Gao XD, Bi-Jie H, et al. Risk factors of mortality in patients with bloodstream infections due to Acinetobacter baumannii. Chin J Nosocomio, 2017, 27 (12): 2668-2671.

[13] Gu Z, Han Y, Meng T, et al. Risk Factors and Clinical Outcomes for Patients With Acinetobacter baumannii Bacteremia. Medicine (Baltimore). 2016; 95 (9): e2943.

[14] Lee YT, Chiang MC, Kuo SC, et al. Carbapenem Breakpoints for Acinetobacter baumannii Group: Supporting Clinical Outcome Data from Patients with Bacteremia. PLoS One. 2016; 11 (9): e0163271. Published 2016 Sep 19.

[15] Liu CP, Shih SC, Wang NY, et al. Risk factors of mortality in patients with carbapenem-resistant Acinetobacter baumannii bacteremia. J Microbiol Immunol Infect. 2016; 49 (6): 934-940.

[16] Guo N, Xue W, Tang D, Ding J, Zhao B. Risk factors and outcomes of hospitalized patients with blood infections caused by multidrug-resistant Acinetobacter baumannii complex in a hospital of Northern China. Am J Infect Control. 2016; 44 (4): e37-e39.

[17] Freire MP, de Oliveira Garcia D, Garcia CP, et al. Bloodstream infection caused by extensively drug-resistant Acinetobacter baumannii in cancer patients: high mortality associated with delayed treatment rather than with the degree of neutropenia. Clin Microbiol Infect. 2016 Apr; 22 (4): 352-358.

[18] Long Y, Guo QH, Liu DW, et al. Risk Factors and Clinical Outcomes of Pan-drug Resistant Acinetobacter baumannii Bacteremia. Med J PUMCH, 2015, 6 (04): 260-266.

[19] Nutman A, Glick R, Temkin E, et al. A case-control study to identify predictors of 14-day mortality following carbapenem-resistant Acinetobacter baumannii bacteraemia. Clin Microbiol Infect. 2014; 20 (12): O1028-O1034.

[20] Park SY, Choo JW, Kwon SH, et al. Risk Factors for Mortality in Patients with Acinetobacter baumannii Bacteremia. Infect Chemother. 2013; 45 (3): 325-330.

[21] Kim SY, Jung JY, Kang YA, Lim JE, Kim EY, Lee SK, et al. Risk factors for occurrence and 30-day mortality for carbapenem-resistant Acinetobacter baumannii bacter- emia in an intensive care unit. J Korean Med Sci 2012; 27: 939-47.

[22] Esterly JS, Griffith M, Qi C, et al. Impact of carbapenem resistance and receipt of active antimicrobial therapy on clinical outcomes of Acinetobacter baumannii bloodstream infections. Antimicrob Agents Chemother. 2011; 55 (10): 4844-4849.

[23] Song JY, Cheong HJ, Choi WS, et al. Clinical and microbiological characterization of carbapenem-resistant Acinetobacter baumannii bloodstream infections. J Med Microbiol. 2011; 60 (Pt 5): 605-611.

[24] Anunnatsiri S, Tonsawan P. Risk factors and clinical outcomes of multidrug-resistant Acinetobacter baumannii bacteremia at a university hospital in Thailand. Southeast Asian J Trop Med Public Health. 2011; 42 (3): 693-703.

[25] Routsi C, Pratikaki M, Platsouka E, et al. Carbapenem-resistant versus carbapenem-susceptible Acinetobacter baumannii bacteremia in a Greek intensive care unit: risk factors, clinical features and outcomes. Infection. 2010; 38 (3): 173-180.

[26] Tseng YC, Wang JT, Wu FL, Chen YC, Chie WC, Chang SC. Prognosis of adult patients with bacteremia caused by extensively resistant Acinetobacter baumannii. Diagn Microbiol Infect Dis. 2007; 59 (2): 181-190.

[27] Choi JY, Park YS, Kim CO, et al. Mortality risk factors of Acinetobacter baumannii bacteraemia. Intern Med J. 2005; 35 (10): 599-603.

[28] He S, Li Z, Yang Q, Quan M, Zhao L, Hong Z. Resistance Trends Among 1,294 Nosocomial Acinetobacter baumannii Strains from a Tertiary General Hospital in China, 2014 - 2017. Clin Lab. 2020; 66 (3): 10.7754/Clin.Lab.2019.190629.

[29] Knaus WA, Draper EA, Wagner DP, Zimmerman JE. APACHE II: a severity of disease classification system. Crit Care Med. 1985; 13 (10): 818-829.

[30] Uwingabiye J, Lemnouer A, Baidoo S, et al. Intensive care unit-acquired Acinetobacter baumannii infections in a Moroccan teaching hospital: epidemiology, risk factors and outcome. Germs. 2017; 7 (4): 193-205.

[31] Xiao D, Wang L, Zhang D, Xiang D, Liu Q, Xing X. Prognosis of patients with Acinetobacter baumannii infection in the intensive care unit: A retrospective analysis. Exp Ther Med. 2017; 13 (4): 1630-1633.

[32] El Mekes A, Zahlane K, Ait Said L, Tadlaoui Ouafi A, Barakate $\mathrm{M}$. The clinical and epidemiological risk factors of infections due to multi-drug resistant bacteria in an adult intensive care unit of University Hospital Center in Marrakesh-Morocco. J Infect Public Health. 2020; 13 (4): 637-643. 